\title{
Actividad del sistema de defensa antioxidante y agresión oxidativa en eritrocitos de recién nacidos de bajo de peso y distintas edades gestacionales
}

\author{
B.Q. Patrieio Cañas D. ${ }^{1}$; Dr. Manuel Olivares G. ${ }^{2}$ Sra. Consuelo Celedón G. ${ }^{3}$; \\ T.M. Sandra Llaguno $\mathrm{A}^{2}{ }^{2}$; B.Q. Alfonso Valenzuela B. ${ }^{\text {' }}$
}

\section{Antioxidant enzime defense system in cord blood from infants}

\begin{abstract}
Finzyme activity of antioxidant defense system and oxidative stress have becn deteminod in cord blood of 59 specimens obtained from infants with low birth weight and over a range of gestational ages. Three diflerent enzyme activities of this system have been determined, that is, superoxide dismutase, catalase, and glutathion peroxidase. There are not significant differences in the activity of these enzymes. However, the levels of malonyldialdehyde (MDA), a product of lipid peroxidation. are significantly higher in preterm newborns compared with term newborns. The physiological implications of these results, specially related witl fetal erythrocy tes behaviour at different gestational ages and its relation with oxygen therapy are discussed.

(Key words: lipid pefoxidation, gestationa! age, premature infants).
\end{abstract}

El oxigeno es fundamental para la sobrevida de los organismos aeróbicos. Sin embargo, en ciertas condiciones ha sido asociado con efectos tóxicos de algunos tejidos, produciendo displasia bronco pulmonar ${ }^{1.4}$ y fibroplasia retrolental ${ }^{5}$ enfermedades que son características de los riños de pretérmino.

Para protegerse contra el daño producido por la presencia de radicales libres del oxígeno que se producen al reaccionar $\mathrm{O}_{2}$ con la hemoglobina o cualquier otra metalo-proteína, el organismo tiene un eficiente sistema de defensa antioxidante, especialmente activo en el eritrocito. Se ha encontrado independientemente que existen altos niveles de catalasa ${ }^{6}$ y relativamente menores niveles de glutatión peroxidasa ${ }^{7}$ y superóxido dismutasa ${ }^{8}$ en eritrocitos, enzimas que pueden metabolizar agua oxige-

1. Unidad de Bioquímica Farmacológica. INTA. Universidad de Chile.

2. Unidad de Hematología. INTA. Universidad de Chile.

3. Egresada de la Facultad de Química y liarmacia. Universidad de Chilc. nada $\left(\mathrm{H}_{2} \mathrm{O}_{2}\right)$ y metabolitos activos del oxígeno como el radical libre superóxido $\left(\mathrm{O}_{2}-\right)$ y el radical libre hidroxilo $(\cdot \mathrm{OH})$.

Dado que el eritrocito es una célula con una alta especialización bioquímica, como es el transporte de oxígeno a los tejidos, nosotros nos interesamos en medir el nivel de actividad de estas tres metaloenzimas relacionadas con el sis. tema de defensa antioxidante en niffos de bajo peso de nacimiento y de distinta edad gestacional, con el objeto de observar si existen cambios significativos con respecto a estas variables.

Estos resultados pueden tener importancia además, para explicar el comportamiento fisio. lógico de distintos tipos de eritrocitos y relacionarlos con la edad gestacional. En este sentido, se conoce que los eritrocitos de los niños de término tiene una vida media mayor que los eritrocitos de niños prematuros, esto es, se considera que el proceso de deterioro orgánico es mayos en eritrocitos de niños de menor edad gestacional9,10. Uno de los factores que puede influir en el acortamiento de la vida media del eritrocito son los diferentes niveles de lipoperoxidación que en él se producen. ${ }^{11,12}$ Para evaluar estos distintos 
Tabla 1

Distribución de los niños por cdad gestacional y peso al nacer (P.N.)

\begin{tabular}{|c|c|c|}
\hline Grupo I: & $\begin{array}{l}\text { Edad gestacional } \\
37 \text { semanas o mas } \\
\text { Adecuados } n=7 \\
\text { Pequeños } n=12\end{array}$ & $\begin{array}{l}\text { Promedio P.N. } \\
2330 \pm 41 \\
2302 \pm 107\end{array}$ \\
\hline Grupo II: & $\begin{array}{l}\text { Edad gestacional } \\
35-36 \text { semanas } \\
\text { Adecua dos } n=9 \\
\text { Pequeños } n=10\end{array}$ & $\begin{array}{l}2246 \pm 299 \\
1936 \pm 264\end{array}$ \\
\hline Grupo IsE: & $\begin{array}{l}\text { Edad gestacional } \\
33-34 \text { semanas } \\
\text { Adecuados } n=13 \\
\text { Pequeños } n=10\end{array}$ & $\begin{array}{l}1950 \pm 250 \\
1892 \pm 180\end{array}$ \\
\hline
\end{tabular}

P.N.: Peso al nacer ( $\ddot{\mathrm{X}} \pm$ D.E. $)$

retos oxidativos, se midieron los niveles eritrocitarios de malondialdehido (MDA), un producto de la lipoperoxidación. Los resultados de esta investigación se discuten de acuerdo a algunos hallazgos fjsiológicos que nosotros previamente hemos encontrado en el laboratorio, con respecto al distinto comportamiento prooxidante de la hemoglobina fetal humana (HbF) comparada con hemoglobina adulta $(\mathrm{HbA}) .^{13}$

\section{MATERIAL Y METODO}

Se tomó sangre del cordón umbilical de 59 niños de distinta edad gestacional provenientes de la materuidad del Hospital Paula Jaraquemada en tubos que contienen EDTA $0,1 \mathrm{mg} / \mathrm{mL}$ de sangre. Los eritrocitos se separaron del plasma por centrifugación $y$ se lavaron tres veces con solución de cloruro de sodio 9\%/00. Las actividades de las enzimas y la agresión oxidativa se midieron en distintas alicuotas de eritrocitos de acuerdo a los métodos que se describen posterjormente. Los niños que se utilizar on para hacer estas mediciones tenía un peso de nacimiento menor de $2.500 \mathrm{~g}$. Se excluyeron de erte estudio todos los niños que presentaron infección, incompatibilidad del grupo $\mathrm{Rh}, \mathrm{ABO}$, dificulted respiratoria y malformaciones graves.

La edad gestacional de los recién nacidos se determinó de acuerdo al método de Dubowitz y col.14

Los niños a su vez se clasificaron como pequeños y adecuados para la edad gestacional de acuerdo a las tablas de Lubchenko. 15

Se tomaron tres grupos de niffos con distinta edad gestacional que se subciasificaron como adecuados y pequeños para la edad gestacional, como describe en la tabla 1 .

Las enzimas'se midieron tomando alicuotas de la suspensión de erítrocitos de acuerdo a métodos previamerite descritos en la literatura y realizados en el laboratorio. Para determinar la actividad de la cuproenzima superóxido dismutasa se uttlizá el método Winter. bourn y col. 16 como se describió anteriormente. $17 \mathrm{La}$ enzima catalasa fue medida de acuerdo al método de la cinćtica de utilización de NADPH. 19

Los niveles de lipoperoxidación o agresión oxidativa del eritrocito se midier on determinando el malondialdehido (MDA) formado, con el método de Fee $y$ Teitelbaum. 20

Todas las mediciones se expresaron en unidades o umoles por gramo de hemoglobina (gHb). La hemoglobina se determinó de acuerdo al método de Drabkin. 21

Para determinar si las diferencias eran significativas entre los distintos grupos de edad gestacional y peso al nacer estudiados se usó la prueba de Student para datos no pareados, 22

\section{RESULTADOS}

La tabla 2 muestra la actjidad de las tres

Tabla 2

Actividad de enzimas relacionadas con el sistema de defensa antioxidante en niưos de distin ta cdad gestacional (EG) y peso al nacer (PN).
U. CAT
U. GASH Px
U. SOD

Grupo I

Adecuado $11=7$

$105,9 \pm 43,1$

$1,25 \pm 0,25$

$2.307 \pm 293,0$

Pequenton $=12$

$138,8 \pm 47,2$

$1,49 \pm 0,52$

$2.358 \pm 673,0$

Grupo I]

Adecuado $\mathrm{n}=9$

$78,44 \pm 23,3$

$1,40 \pm 0,35$

$1.512 \pm 672,2$

Pequeñon $\mathrm{n}=8$

$128,6 \pm 44,9$

$1,68 \pm 0,46$

$1.806 \pm 850,4$

Grupo UI

Adecuado $n=\$ 3$

$133,8 \pm 60,5$

$1,80 \pm 0,41$

$1.597 \pm 685,0$

Pequeño $n=10$

$$
87,3 \pm 42,6
$$

$1,46 \pm 0,27$

$1.917 \pm 439,6$ 
Tabla 3.

Concentración de MDA umoles/gHb ( $\overline{\mathrm{X}} \pm$ D.E.) en distintos grupos de edad gestaciona] (EG) $y$ peso para la misma

\begin{tabular}{lccc}
\hline Peso al nacer & Grupo I & Grupo Il & Grupo III \\
& & & \\
Adecuado (a) & $0,11 \pm 0,02^{+*}$ & $0,26 \pm 0,7^{+}$ & $0,31 \pm 0,07^{*}$ \\
Pequeño (p) & $0,08 \pm 0,02^{+}$ & $0,20 \pm 0,11$ & $0,28 \pm 0,11^{+}$ \\
\hline
\end{tabular}

Los valores de $p 0,05$ fueron considerados significativos.

+ Grupo la vs Grupo Ila p $<0,02$

Giupo Ip ys Grupo III $\mathrm{p}<0,01$

* Grupo Ia vs Grupo Illa p $<0,05$

cnzimas estudiadas (catalasa, superóxído dismu. tasa y glutation peroxidasa) expresadas como unidades por $\mathrm{gHb}$. No hay diferencias significativas entre los distintos grupos de edad gestacional y tamaño para las mismas. Tampoco existen diferencias dentro de los mismos grupos según el peso al nacer. Por otra parte, como una forma de evaluar la diferente agresión oxidativa a que están expuestos los eritrocitos, midieron los niveles de MDA (tabla 3) expresados en moles/gHb. En estas determinaciones encontraron diferencias significativas entre los niños de preténnino

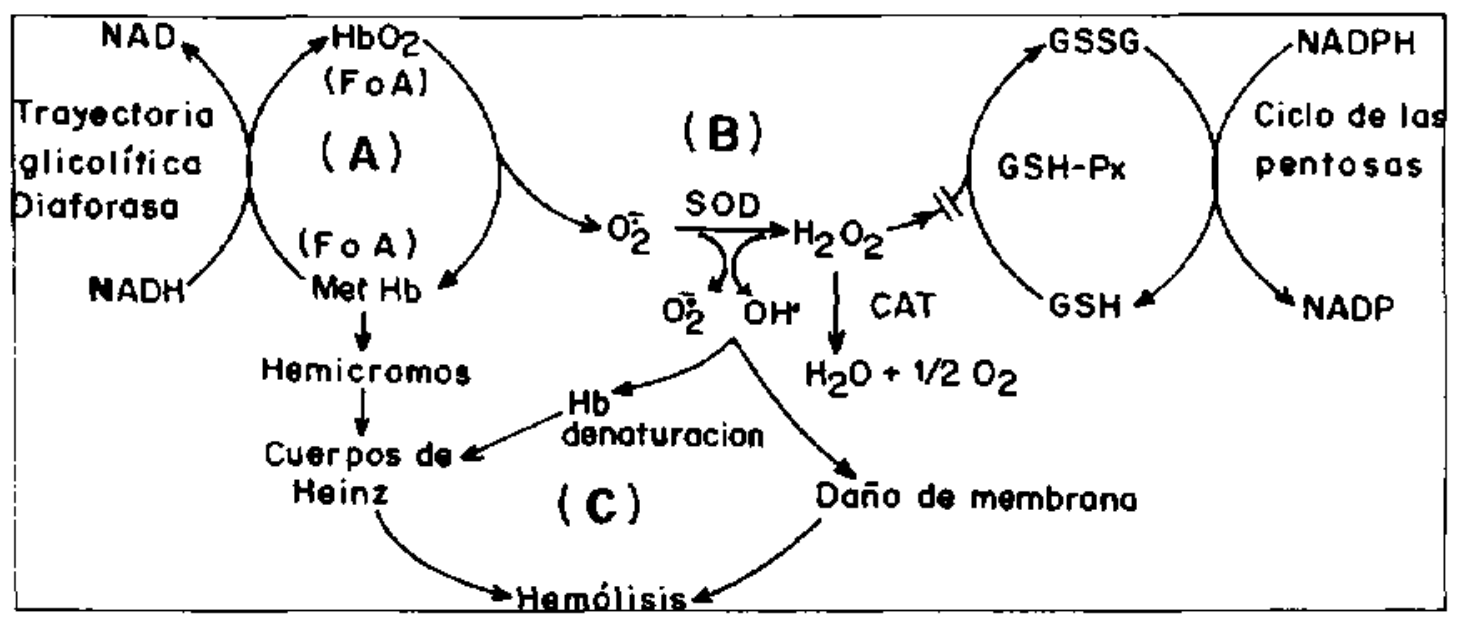

Figura: Mecanismos de defensa antioxidante en critrocitos fetales y adultos:

A) Autooxidante de la hemoglobila (Hb) fetal (F) o adulta (A) human genera metahemogiobina (MetHb) y radicales libres de oxígeno $\left(\mathrm{O}_{2}\right)$ que pueden ser metabolizados por diferentes enzimas del sistema de defensa antioxidante.

La MetHb formada a su vez pucde ser transformada por la enzima diaforasa y NADH formado en la trayectoria glicolitica a oxihemoglobina $\left(\mathrm{HbO}_{2}\right)$.

B) Sistema de defensa antioxidante formado por superóxido dismutasa (SOD), catalasa (CAT), glutatión peroxidasa (GSH-Px) que requicre glutatión reducido (GSH) y la forma de glutatión oxidado (GSSG) que puede ser metabolizado por enzimas del ciclo de las pentosas en presencia de NADPH y regenerar glutatión reducido (GSH).

C) La acumulación de especies de oxígeno activado por la ausencja de antioxidantes o por disminuclón de la actividad del sistema de defensa antioxidantc puede producir acumulación de especies activas del oxigeno $\left(\mathrm{O}_{2} ; \mathrm{OH}^{\circ}\right)$ que pueden producir daño de la membrana o denaturación de la hemoglobina con formación de cuerpos de Heirz, factores qui puedencausar hemólisis. 
(Grupo II y Grupo III) con respecto a los niveles de MDA de los niños de término (Grupo I). Sin embargo, no hay diferencias significativas en un mismo grupo entre los niños clasificados como de peso gestacional adecuado con respecto a los niños de peso pequeño al nacer.

Estos resultados indican que hay una diferencia significativa en los niveles de agreșión oxidativa entre los niños de pre término y los de tếrmino, siendo mayor la agresión oxidativa a medida que disminuye la edad gestacional.

\section{DISCUSION}

La importancia de los resultados descritos anteriormente puede entenderse mejor al considerar la relación que existe entre los diferentes formas parcialmente reducidas del oxígeno y las enzimas relacionadas con el sistema de defensa ant ioxidante, como se describe en la figura.

Según la figura 1 el paso de oxi $\mathrm{Hb}\left(\mathrm{Fe}^{+2}\right)^{\prime}$ a meta $\mathrm{Hb}\left(\mathrm{Fe}^{+3}\right)$ puede generar radicales superóxidos $\left(\mathrm{O}_{2}\right)$ que pueden generar a su vez el radical libre de hidroxito y agua oxigenada $\left(\mathrm{H}_{2} \mathrm{O}_{2}\right)$, compuestos que a su vez pueden dañar las membranas y otros componentes celulares. La generación de radicales superóxidos, durante la autooxidación de la hemoglobina, fue inicialmente demostrada en los trabajos de Misra y Frido. vich. ${ }^{23}$

Así también, se ha encontrado una disminución de la actividad de algunas de las enzimas del sistema de defensa antioxidante en algunas patologías como las anemias de Fanconi. ${ }^{24}$ Las alte. raciones provocadas por la generación de estos radicales libres del oxígeno en daños producidos en la membrana del glóbulo rojo ha sido estudiada por Jain ${ }^{25,26}$ en pacientes con anemia falciforme. Los resultados encontrados en la tabla con respecto a los mayores niveles de malondialdehido formado en eritrocitos de niños de pretérmino (Grupo II y III) comparado con los niveles de MDA de los niffos de término (Grupo I), pueden indicar que existen diferencias en la capacidad de generar radicales superóxidos entre anbos eritrocitos, a pesar de tener una misma dotación de enzimas del sistema de defensa antioxidante (tabla 3 ).

Una posible explicación de este hecho puede residir en la diferente proporción de $\mathrm{HbF}$ con respecto a HbA durante la gestación, como fue informado por Bard. ${ }^{27}$ Nosotros hemos demostrado recientemente en el laboratorio que ambas hemoglobinas humanas tienen una diferente capacidad prooxidante en su estado de oxihemoglobina. ${ }^{13}$ Esta hipótesis puede ser cierta ya que la relación entre ambas hemoglobinas ( $\mathrm{HbF} / \mathrm{HbA}$ ) disminuye en forma proporcional durante la ges. tación, siendo mayor en los niños de pretérmino (Grupo II y Grupo III) con respecto a los niños de término (Grupo I).

Otro factor que puede contribuir a la mayor cantidad de especies de radicales libres de] oxígeno, además de la relación entre ambas hemoglobinas, es la diferente cantidad de vitamina $E$ ( $V$ it $E$ ) en los niños de término con respecto a los nifíos de pretérmino. ${ }^{28}$

Aunque nosotros no podemos afimmar que existen diferencias en los niveles de esta vitamina en los niños aquí estudiados, es un hecho conocido que la susceptibilidad de los glóbulos rojos se altera por modificaciones nutricionales durante la evolución extrauterina del recién nacido de bajo peso, especialmente, en niños con menos de 1.500 g al nacer. $29-31$

Los resultados de esta investigación aunque no determinan qué factores específicos contribuyen a modificar el estrés oxidativo de los eritrocitos, demuestran que no hay una distinta capacidad del sistema de defensa enzimática de actividad antioxidante, en cuanto a los grupos de distinta edad gestacional y peso al nacer, aquí estudiados.

Los resultados que se obtienen de la literatùra a este respecto son más bien contradictorios y parciales, ya que generalmente sólo se refieren a los niveles de una de las enzimas y además están sujetos a diferentes tipos de variaciones, ${ }^{32,33} \mathrm{Sin}$ embargo, los resultados informados aquí se parecer a aquellos informados por Autor y col, ${ }^{34}$ quien no encuentra que existan diferencias im. portantes en los niveles de su superóxido dismutasa.

El valor de esta investigación, con respecto a los datos obtenidos anteriormente de la literatura, radica en que en este estudio se midió el nive] de las tres enzimas más importantes del sistema de defensa antioxidante simultăneamente, en ni. for de bajo peso al nacer, donde existe un mayor riesgo de contraer algunas enfermedades que pueden requerir $\mathrm{O}_{2}$-terapia. ${ }^{35}$ Considerando estos resultados creemos que es necesario hacer estudios con niños de menor edad gestacional, en los cuales la deficiencia de Vitamina E, los hace 
más susceptibles de contraer ciertas patologías relacionadas con agresión oxidativa. ${ }^{36}$

\section{RESUMEN}

Se analizaron en este estudio muestras de sangre de cordón umbilical de 59 niños de bajo peso al nacer y distinta edad gestacional, obtenidas de la Maternidad del Hospital Paula Jaraquemada. No se encontraron diferencias significativas en la actividad de superóxido dismutasa, catalasa y glutatión peroxidasa en estos niños con respecto a edad gestacional y peso al nacer. Los eritrocitos de los niños de pretérmino tezían, sin embargo, un mayor nivel de malondialdehido (MDA) que los eritrocitos de los niños de término. Esto puede determinar que los eritrocitos están expuestoş a distinta agresión oxidativa de acuerdo a la edad gestacional. Más estudios son necesarios para establecer una relación de estos resultados y el comportamiento físiológico de los eritrocitos a distinta edad gestacional y la rela. ción de estos hechos con la etiología de distintos tipos de anemia que sufren estos niños después de nacer.

\section{AGRADECIMIENTOS}

Se agradece la asistencia técnica del Sr. Ricardo Guerra quién hizo las determinaciones de enzinas $y$ el manejo de las muestras.

Esti trabajo fue financiado por la Universidad de Clike, Proyecto DIE B-1399-8613.

\section{REFERENCIAS}

1. Autor A.P., Roberts R.J.: Superoxide dismutase activity and isoenzynes in the human fetal and adult Jung. Fed. Proc., 1974; 33: 1505-1510.

2. Northway W.H. Rosen R.C. Porter D. Y.: Pulmonary disease following respiratory therapy of hyaline membranc disease, New Fngl J Med 1967; 276: 357-358.

3. Roberts R, Frank $L_{\text {, }}$ Autor A.: Superoxide dismutase: An enzyme of maturation - Relationship of IRDS. Pediatr Res 1975;9: 136-139.

4. Crapo J.D. and Tierney D.F.: Superoxide dismutase and pulmonary oxygen toxicity. Amer J Physiol $1974 ; 225$ : 1404-1408.

5. James L.S., and Lanmon S.T. Ed.: History of oxygen therapy and retrolental fibroplasia. Pediatrics 1976: 57: 591-595.

6. Aebi H., Bossi E, Cantz M., Matsubara S., Suter H, A catalasemia in Switzerland. En: Hereditary Disorders of Erythrocyte Metabolism (Ld). Beutler, E. pp. 41-65 (1968).

7. Cohen G., Hochstein P.: Glutathone peroxidast: the primary agent for the elimination of hydrogen peroxide in erythrocytes. Biochemistry 1963; 2: 1420-1426.

8. Lynch R.E., Lee G.R., Cartwright GE.: Inhibition of superoxide dismutase of methemoglobin formation from oxyhemoglobit. J Biol Chem 1976: 251: 1015-1018.

9. Pearson H.A.: Life span of the fetal red blood cell. J Pediatr 1967; 70: 166-168.

10. Bratteby L.E.: Studies on ery throkinetics in infancy. Acta Paediatr Scand 1968; 57:311-320.

11. Valencuela $A$, Videla L.A.: Citotoxicidad de1 oxígeno, aspectos biológicos y médicos. Rev Med Chile 1983; 111 : 829-836.

12. Fridovich $1 .:$ The biology of oxygen radicals. Science 1978; 201: 875-876.

13. Valenzuela A., Guerra R., Lazcano L., Cañas $P$.: Differential prooxidative effects of adult and fetal hemoglobin. Febs. Letters 1986; 196: 353-356.

14. Dubowitz L., Dubowisz W., Goldberg C.: Clinical assessment of gestational age in the newborn infant. J Podiat 1970; 77: 1-10.

15. Lubchenko L. O., Honsman C., Pressier M., Boyd E.: Intrauterine growth as cstimated from live born birth weight data at 24 to 42 weeks of gestation Pediatrics $1963 ; 32 ; 793.795$.

16. Wintherboton C., Haekins R., Brain M., Carral R.: The estimation of red cell superoxide dismutase activity. J Lab Clin Med 1975; 85: 337.339.

17. Uauy R., Castillo-Duran C., Fisberg M., Femóndez $N$., Valenzuelo $A .:$ Red cell superoxide dismutase activity as an index of human copper nutrition. J Nutr 1985: 115: 1650-1655.

18. Maral J., Piget $K$., Michelson A.: Comparative sudy of superoxide dismutase, catalase and glutathion peroxidase levels in erythrocytes of different animals. Biochem Biophys Res Comm 1977; 77 : $1525-1535$.

19. Paglia D.E., Vahente H.M.: Studies on the quantitative and qualitative characterization of ery throcyte glutathione peroxidase. J Lab Clin Med 1967; 70: $158-160$.

20. Fee J.A., Teitelboum H.D.: Evidence that superoxide dismutase plays a role in-protecting red blood cells against peroxidative hemolysis. Biochem. Biophys Res Commun 1972; 49: 150-153.

21. International Committe for Standardization in Haematology Recommendations and requirements for haemoglobinometry in human blood. J Clin Path 1965; 16: 353-355.

22. Weber, $E$. En: Grumdiss der Biologischen Statistik, Gustav Fisher Verlag, Ed 1967; 252-258.

23. Misra $H$., Fridovich, $I$ : The generation of superoxide radicals during autooxidation of hemoglobin. $]$ Biol Chem 1972; 247: 6960-6962.

24. Mavelli I., Ciriolo M.R., Rotilio G.: Superoxide dismutase, glutathione peroxidase, and catalasc in oxidative hemolisis. A study of Fanconi's anemia ery throcytes. Biochem Biophys Res Commun 1982; 106: 286-290.

25. Jain S.K., Shohet S.B.: A novel phospholipid in irreversibly sickled cells; evidence for in vivo peroxidative membrane damage in sickle cell disease. Blood 1984; 63: 362-367.

26. Jain S.K., Mohantas S.M., Clatk M., Sholet, S.B. 
The effect of malondialdehyde, a protuct of lipid peroxidation, on the deformability, dehydration and $5{ }^{1} \mathrm{Cr}$-aurviral of ery throcytes Brit J Haematol $1983 ; 53: 247-255$.

27. Bard H.: Postnatal fetal and adult hemoglobin synthesis in early preterm newborn infants. J. Clin Invest 1973;52:1789-1795.

28. Gross $S$ : Hemoly tic anemic in prematare infants: relationship to vitamin E. Selenium and glutathion peroxidase and erythrocy te lipis. Seminars in Hematology $1976 ; 13: 187-201$.

29. Hassan H., Hashim S.A., Van Itallie T.B.: Syndrome in premature infants associate with low plasma Vitanin E levels and high polyunsaturated fatty acid diet. Am J Clin Nutr 1966; 19: 147.151.

30. Oski I.A., Bormess L.A.: Vitamin E deficiency: A pteviously unrecognized cause of hemolytic anemia in the premature infant. J Pedjatt 1967; 70: 211-214.

31. Zipurski A.: Vitamin E deticiency anemia in newbort infants. Clin Perinetalogy 1984; 11: $393-402$.
32. Salk L.A., Hsieh H. Baricos W.H., Shapira, E. Enzymatic and immunological quantitation of erythrocyte superoxide dismutase in adults and in neonates of different gestational ages. Pediatr Res $1982 ; 16: 933-936$

33. Bonta B.W., Gowron E.R., Worshaw J.B.: Neonatal rod cell superoxide dismutase enzyme levels: Possible role as a cellular defense mechanism against pulmonary oxygen toxicity. Pediatr Res 1977; 11: 754-757.

34. Autor A.P., Frank L., Roberts R.T.: Developmental characteristics of pulmonary. Superoxide dismutase: Relationship to idiopathic respiratory distress syndrome. Pediatr Res 1976; 10: 154-158.

35. Frank L., Groseclose F.: Oxygen toxicity in newborn rats: the adverse effects of undemutrition. 5 Appl Physiol Respirat Enviton. Exercise Physio? $1982 ; 53 ; 1248-1255$.

36. Cynamon H,A., Isenberg J.M., Mgufen Co. H.: Erythrocyte malonmondialdehy de release in vitro: a functional measure of Vitamin E Status. Clin Chim Acta 1985; $151: 169.176$. 